\section{Exercise for Mental Health}

Sir: In this era of exponential growth of the "metabolic syndrome" and obesity, lifestyle modifications could be a cost-effective way to improve health and quality of life. Lifestyle modifications can assume especially great importance in individuals with serious mental illness. Many of these individuals are at a high risk of chronic diseases associated with sedentary behavior and medication side effects, including diabetes, hyperlipidemia, and cardiovascular disease. ${ }^{1}$ An essential component of lifestyle modification is exercise. The importance of exercise is not adequately understood or appreciated by patients and mental health professionals alike. Evidence has suggested that exercise may be an oftenneglected intervention in mental health care. ${ }^{2}$

Aerobic exercises, including jogging, swimming, cycling, walking, gardening, and dancing, have been proved to reduce anxiety and depression. ${ }^{3}$ These improvements in mood are proposed to be caused by exercise-induced increase in blood circulation to the brain and by an influence on the hypothalamic-pituitaryadrenal (HPA) axis and, thus, on the physiologic reactivity to stress. ${ }^{3}$ This physiologic influence is probably mediated by the communication of the HPA axis with several regions of the brain, including the limbic system, which controls motivation and mood; the amygdala, which generates fear in response to stress; and the hippocampus, which plays an important part in memory formation as well as in mood and motivation.

Other hypotheses that have been proposed to explain the beneficial effects of physical activity on mental health include distraction, self-efficacy, and social interaction. ${ }^{4}$ While structured group programs can be effective for individuals with serious mental illness, lifestyle changes that focus on the accumulation and increase of moderate-intensity activity throughout the day may be the most appropriate for most patients. ${ }^{1}$ Interestingly, adherence to physical activity interventions in psychiatric patients appears to be comparable to that in the general population.

Exercise improves mental health by reducing anxiety, depression, and negative mood and by improving selfesteem and cognitive function. ${ }^{2}$ Exercise has also been found to alleviate symptoms such as low self-esteem and social withdrawal. ${ }^{3}$ Exercise is especially important in patients with schizophrenia since these patients are already vulnerable to obesity and also because of the additional risk of weight gain associated with antipsychotic treatment, especially with the atypical antipsychotics. Patients suffering from schizophrenia who participated in a 3-month physical conditioning program showed improvements in weight control and reported increased fitness levels, exercise tolerance, reduced blood pressure levels, increased perceived energy levels, and increased upper body and hand grip strength levels. ${ }^{5}$ Thirty minutes of exercise of moderate intensity, such as brisk walking for 3 days a week, is sufficient for these health benefits. Moreover, these 30 minutes need not to be continuous; three 10-minute walks are believed to be as equally useful as one 30 -minute walk.

Health benefits from regular exercise that should be emphasized and reinforced by every mental health professional to their patients include the following:
1. Improved sleep

2. Increased interest in sex

3. Better endurance

4. Stress relief

5. Improvement in mood

6. Increased energy and stamina

7. Reduced tiredness that can increase mental alertness

8. Weight reduction

9. Reduced cholesterol and improved cardiovascular fitness

Mental health service providers can thus provide effective, evidence-based physical activity interventions for individuals suffering from serious mental illness. Further studies should be done to understand the impact of combining such interventions with traditional mental health treatment including psychopharmacology and psychotherapy.

The authors report no financial or other affiliation relevant to the subject of this letter.

\section{REFERENCES}

1. Richardson CR, Faulkner G, McDevitt J, et al. Integrating physical activity into mental health services for persons with serious mental illness. Psychiatr Serv 2005;56:324-331

2. Callaghan P. Exercise: a neglected intervention in mental health care? J Psychiatr Ment Health Nurs 2004;11:476-483

3. Guszkowska M. Effects of exercise on anxiety, depression and mood [in Polish]. Psychiatr Pol 2004;38:611-620

4. Peluso MA, Andrade LH. Physical activity and mental health: the association between exercise and mood. Clinics 2005;60: $61-70$

5. Fogarty M, Happell B, Pinikahana J. The benefits of an exercise program for people with schizophrenia: a pilot study. Psychiatr Rehabil J 2004;28:173-176

Ashish Sharma, M.D.

University of Nebraska Medical Center Vishal Madaan, M.D.

Creighton University/

University of Nebraska Medical Center

Frederick D. Petty, M.D., Ph.D.

Omaha VA Medical Center

Omaha, Nebraska

\section{Citalopram Treatment for Inappropriate Sexual Behavior in a Cognitively Impaired Patient}

Sir: Sexual inappropriateness and hypersexuality can be defined as vigorous sexual drive or other sexually related problems that interfere with normal activities of daily living, or sexual behavior that is pursued at inappropriate times. By definition, such behavior is persistent, uninhibited, and directed against oneself or toward unwilling partners. Inappropriate sexual behavior encompasses a range of behavior, including suggestive language, flirtation, fondling, removing one's clothing, and masturbating in public. The rates of sexual disinhibition reported in the literature in people diagnosed with Alzheimer's disease living both in the community and in residential care range from $2.9 \%$ to $8 \%$. $^{1}$ These behaviors may result from mental and physical illnesses, alone or in combination. ${ }^{2}$

Work has been done as to how hospital and nursing home staff should respond to these behaviors and guide 
the development of management strategies and care plans. Most researchers agree that behavioral, psychological, and environmental interventions are preferable to the risks of pharmacology. ${ }^{1}$ However, Harris and Wier, ${ }^{3}$ in a review of the literature, found that, in many cases, pharmacologic treatment is often the preferred first-line treatment for hypersexual behavior because of its ease of administration, perceived efficacy, and decreased use of staff time. Numerous medications have been tried for the treatment of such behaviors, but there are no convincing data supporting the use of a particular medication. Because most evidence is in the form of case reports, data are also lacking regarding the advantage of any medication over placebo or in comparison with other medications.

Some preliminary reports indicate that selective serotonin reuptake inhibitors (SSRIs) might be effective in controlling such behaviors. ${ }^{4,5}$ The reason for their effectiveness has yet to be established, but the effectiveness could be due to their antiobsessive and antilibidinal effects. To our knowledge, no case reports have been published on the use of citalopram for such behaviors. We report successful use of citalopram in the treatment of inappropriate sexual behavior in a cognitively impaired adult with a history of bipolar disorder.

Case report. Mr. A, a 54-year-old man with a long history of bipolar disorder and recent onset of cognitive deficits due to Parkinson's disease, had been displaying inappropriate sexual behavior on and off for the last 5 years. This problem had recently become worse and had necessitated multiple hospital admissions. At the time of this admission, he was on a regimen that included lamotrigine, clozapine, aripiprazole, ziprasidone, and olanzapine. Once he was admitted, all of his psychotropic medications except clozapine were stopped and lithium $150 \mathrm{mg}$ b.i.d. was initiated; after a few days, clozapine was stopped as well.

Mr. A continued to be disorganized and was making sexually inappropriate comments and touching female staff members, which progressed to making inappropriate comments toward male peers and staff members. This behavior had not responded to the numerous antipsychotic medications he was taking at the time of this admission. Estrogen, which has been considered effective in reducing inappropriate sexual behavior, seemed only to have attenuated this behavior and caused the patient to develop gynecomastia, which was unacceptable to him and his family.

Mr. A was difficult to interview; he was disorganized and extremely distractible. During his lucid periods when he had more insight, however, he would talk about thoughts of a sexual nature that he was unable to get rid of despite identifying them as improper. Neither could he control the urges to touch female companions or make comments of a sexual nature.

After the behavior had been observed by the treatment team for about a month, during which time trials to manage the patient behaviorally had failed, treatment with citalopram $20 \mathrm{mg} /$ day was started. Five days after the start of citalopram, Mr. A's inappropriate sexual behavior started to disappear. Two weeks into therapy, although he remained somewhat disorganized in his thoughts, the sexual inappropriateness had disappeared. No side effects were reported by the patient or noted by the treatment team. There were no concomitant medication changes.

In this case, we postulate that the sexually disinhibited behavior was caused by obsessive thoughts and resulted in compulsive behavior. One can speculate that the beneficial effects of SSRIs on these behaviors can at least partially be explained by the effectiveness of this class of medications in treating obsessive-compulsive spectrum disorders. Another possibility is that, in some patients, SSRIs might have the side effect of diminishing libido. However, it has also been reported that SSRIs cause disinhibition of libido. ${ }^{6}$

Of note, this particular patient has Parkinson's disease, a frequent cause of such behavior. Interestingly in our case, such behavior could not have been related to dopaminergic treatment since no such treatment was used. This finding raises the possibility that some of the inappropriate sexual behavior that patients with Parkinson's disease display is not the result of dopaminergic drugs but rather may be a consequence of dementia which itself often accompanies this disease.

SSRIs - in our case, citalopram — might be of use in treating inappropriate sexual behavior. Controlled trials are needed to establish the efficacy of these agents in treating such behavior. If efficacy is established, further investigation to clarify why these medications are so effective is warranted.

The authors report no financial or other affiliation relevant to the subject of this letter:

\section{REFERENCES}

1. Higgins A, Barker P, Begley CM. Hypersexuality and dementia: dealing with inappropriate sexual expression. Br J Nurs 2004;13: 1330-1334

2. Lesser JM, Hughes SV, Jemelka JR, et al. Sexually inappropriate behaviors: assessment necessitates careful medical and psychological evaluation and sensitivity. Geriatrics 2005;60:34-37

3. Harris L, Wier M. Inappropriate sexual behaviour in dementia: a review of the treatment literature. Sex Disabil 1998;16:205-217

4. Levitsky AM, Owens NJ. Pharmacologic treatment of hypersexuality and paraphilias in nursing home residents. J Am Geriatr Soc 1999; 47:231-234

5. Lothstein LM, Fogg-Waberski J, Reynolds P. Risk management and treatment of sexual disinhibition in geriatric patients. Conn Med 1997;61:609-618

6. Greil W, Horvath A, Sassim N, et al. Disinhibition of libido: an adverse effect of SSRI? J Affect Disord 2001;62:225-228

Irakli Mania, M.D. Harun Evcimen, M.D. Maju Mathews, M.D., M.R.C.Psych. Department of Psychiatry Drexel University College of Medicine Philadelphia, Pennsylvania 